\title{
Relación del Bienestar Subjetivo de los trabajadores con su Compromiso hacia la organización
}

\section{The relationship of the Subjective Wellbeing of the Workers with their Commitment towards the Organization}

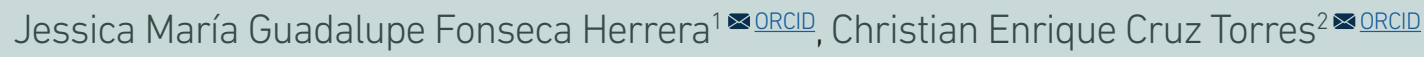 \\ ${ }^{1}$ Instituto Politécnico Nacional \\ 1-2 Universidad de Guanajuato \\ México
}

Fecha correspondencia:

Recibido: mayo 22 de 2018.

Aceptado: enero 16 de 2019.

Forma de citar:

Fonseca Herrera, J.M., \& Cruz

Torres, C.E. (2019). Relación

del Bienestar Subjetivo de los

trabajadores con su Compromiso

hacia la organización. Rev.CES

Psico, 12(2), 126-140.

$\frac{\text { Open access }}{\text { (c) Copyright }}$

Licencia creative commons

Etica de publicaciones

Revisión por pares

Gestión por Open Journal System

DOI: http://dx.doi.org/10.21615/

cesp. 12.2 .9

ISSN: 2011-3080

Sobre los autores:

1. Licenciada en Psicología. Analista de sistemas y procesos en la Coordinación de Enlace

\section{Resumen}

El compromiso organizacional (CO) de los trabajadores es entendido como una actitud o estado de ánimo que deriva en comportamientos como la intención de permanecer en la organización, la identificación con sus valores y la percepción de tener una deuda moral con la misma por los beneficios recibidos. Estudios previos muestran que el CO impacta positivamente el bienestar subjetivo de los trabajadores (BS), entendido este último como la evaluación cognitiva y afectiva que hacen las personas de la satisfacción sobre su propia vida. Otros estudios afirman que es el BS el que predice el CO. El objetivo del presente estudio fue especificar la dirección de esta relación, para lo cual se aplicó el Instrumento de Compromiso Organizacional para evaluar el CO y el módulo básico del Bienestar Autorreportado (BIARE) para evaluar el BS, a 230 trabajadores de una organización educativa pública de nivel superior en México. Mediante modelos de ecuaciones estructurales se contrastaron las hipótesis de relaciones en uno y otro sentido entre ambas variables con una tercera hipótesis con relaciones recíprocas entre ambas. Se identificó como mejor modelo el que presenta relaciones recíprocas y positivas entre el CO y el BS (Chi2=21.16; $\mathrm{gl}=16$; $\mathrm{p}=0.172 ; \mathrm{RMR}=.043 ; \mathrm{GFI}=.976 ; \mathrm{CFI}=.991 ; \mathrm{RMSEA}=0.03$ [IC 90\%=0.00, 0.07]). Se concluye que un incremento en el $\mathrm{CO}$ genera un incremento en el BS de los trabajadores, y la relación positiva entre ambas variables es recíproca. Se discuten las implicaciones de estos resultados para la relación de los trabajadores con su contexto laboral.

Palabras clave: Bienestar Subjetivo, Bienestar en el Trabajo, Bienestar Laboral, Satisfacción Laboral, Compromiso Organizacional, Cultura Organizacional, Clima Organizacional.

\section{Abstract}

The organizational employee commitment conceptualizes the intention to remain in the work place, as the identification of corporative values and the moral sense of engagement due to the benefits received, under the name of organizational commitment (OC). Previous studies show that OC positively 
y Gestión Técnica de la Unidad Profesional Interdisciplinaria de Ingenierías del Instituto Politécnico Nacional, Campus Guanajuato. Profesora del Departamento de Psicología en la Universidad de Guanajuato.

2. Doctor en Psicología. Profesor Asociado al Departamento de Psicología Universidad de Guanajuato. affects the subjective well-being of workers (SW), understood as the cognitive and affective assessment that people make of their own life satisfaction. Other studies state that the effect goes from SW to OC. This study aimed to specify the direction of this relationship, for this purpose OC and SW instruments were applied to 230 workers of a higher education public organization in Mexico. Using structural equation models, the hypothesis of relations in both directions between the two variables was contrasted with a third hypothesis of reciprocal relationships between both. The best model was identified as having positive and reciprocal relationships between $\mathrm{CO}$ and $\mathrm{BS}($ Chi2 $=65,627, \mathrm{df}=18, \mathrm{p}=0.000, \mathrm{RMR}=.074, \mathrm{RMSEA}=.111[\mathrm{Cl} 90 \%=.083, .141]$; $\mathrm{GFI}=.931 ; \mathrm{CFI}=.915)$. It is concluded that increases in OC strengthen $\mathrm{SW}$, and positive relationship between both variables is reciprocal. The implications of the findings on workers' relationships in the work place are discussed.

Keywords: Subjective Well-Being, Well-Being at Work, Labor Well-Being, Job Satisfaction, Organizational Commitment, Organizational Culture, Organizational Climate.

\section{Introducción}

El bienestar de las personas ha sido un tema de interés entre la comunidad académica (Vázquez, Hervás, Rahona, \& Gómez, 2009). Cuestionamientos sobre lo que hace felices a las personas, lo qué significa llevar una buena vida, o los medios y motivos para alcanzar estados de plenitud, son recurrentes en el estudio de la psicología (Abello-Llanos et al., 2009; Díaz et al., 2006; Diener, 1984, 2000; Garaigordobil, Aliri \& Fontaneda, 2009; García, 2002; Larsen \& Eid, 2008). Difícilmente existe una respuesta universal para las cuestiones planteadas, al menos una vigente en diferentes épocas, siendo el bienestar un proceso sensible a los cambios históricos y culturales (Díaz et al., 2006; Diener, 1984; García, 2002; Larsen \& Eid, 2008), toda vez que las evaluaciones que cada individuo realiza de su bienestar resulta de comparaciones entre las expectativas y lo que se tiene, y estas expectativas cambian para diferentes aspectos y etapas de la vida (Diener, Oishi, \& Lucas, 2003; Galinha, Garcia-Martin, Oishi, Wirtz, \& Esteves, 2016).

En consecuencia, existen diversas aproximaciones al concepto de bienestar. Algunos se centran en factores externos como el éxito o la plenitud (Díaz et al., 2006; García, 2002; Vázquez et al., 2009); otros en la evaluación que las personas hacen de su propia vida, o bien en el resultado de un balance entre las emociones positivas y negativas asociadas a la vida diaria (Abello-Llanos et al., 2009; Díaz et al., 2006; Diener, 1984. 2000; García, 2002).

Otra de estas aproximaciones es el bienestar subjetivo (BS), definido por Diener (1984) como la evaluación que hacen las personas sobre sus propias vidas con base en dos componentes, uno cognitivo que comprende la satisfacción con la vida y los diferentes dominios de satisfacción, y otro afectivo. Este último se divide a su vez en afectos positivos, por ejemplo, aquellas emociones de satisfacción por los logros alcanzados o cuando se obtiene el reconocimiento de otras personas por el esfuerzo realizado, y afectos negativos, como las emociones de infelicidad o la molestia generada al recibir una crítica negativa (Diener, 1984, 2000). Al componente afectivo de Diener (1984), otros autores lo han denominado felicidad o balance afectivo, debido a que las personas han de sen8tirse felices en la medida en que sus emociones positivas sean mayores o más frecuentes que las negativas (Dávila \& Jiménez, 2014; Marrero, Carballeira, \& González, 2014; Rojas \& Elizondo-Lara, 2012). 
Pág 128

La evaluación que hacen las personas de su trabajo es muy importante, especialmente si se considera como un medio que permite satisfacer necesidades inmediatas y de socialización. Además, gran parte del bienestar social, material y de la propia vida están profundamente relacionados con las organizaciones y su dinámica (Calderón, Murillo, \& Torres, 2003; González, López-Guzmán, \& Sánchez, 2014; Ochoa, Jacobo, \& Leyva, 2012).
Para autores como Dávila y Jiménez (2014), Marrero et al. (2014) y Rojas y Elizondo-Lara (2012), el BS se refiere a un estado mental positivo que implica una valoración o juicio global de las personas acerca de sus vivencias, logros, fracasos y emociones. Marrero et al. (2014), Dávila y Jiménez (2014) y Diener (1984) coinciden en que el BS está integrado por un componente afectivo y uno cognitivo. Asimismo, Marrero et al. (2014) consideran que el BS está integrado por dos factores: la felicidad y la satisfacción con la vida. La felicidad, entendida como un estado duradero de plenitud que percibe el individuo y que está en función de la evaluación positiva que hace de su entorno, considerando elementos físicos, sociales, afectivos y espirituales de su existencia (Álvarez \& Muñiz, 2013; Marrero et al., 2014). La satisfacción con la vida depende de la evaluación que hace el individuo de áreas relacionadas con la familia, el trabajo y el tiempo libre que tiene disponible (García, 2002; Rojas \& Elizondo-Lara, 2012).

Con independencia de las diferencias entre los modelos teóricos del BS, este concepto se ha convertido en un indicador valioso tanto para los estudiosos de la psicología como para los responsables de las políticas públicas, quienes encuentran en el BS un indicador del éxito global de su administración para mejorar la calidad de vida de la ciudadanía (Diener, Oishi, \& Lucas, 2015).

En tanto que el BS supone una evaluación global de múltiples aspectos de la vida de los individuos y sus entornos, sus niveles son sensibles a factores individuales como la personalidad (Soto, 2015) y la inteligencia emocional (Sánchez-Álvarez, Extremera, \& Fernández-Berrocal, 2016) e incluso por factores genéticos (Okbay et al. 2016). Dentro de los factores externos que afectan el BS se han identificado el apoyo social y familiar (Siedlecki, Salthouse, Oishi, \& Jeswani, 2014), la calidad de la relación de pareja (Carr, Freedman, Cornman, \& Schwarz, 2014) y por supuesto las condiciones laborales y la satisfacción al respecto (Bowling, Eschleman, \& Wang, 2010).

En México, el Instituto Nacional de Geografía e Informática (INEGI, 2016) ha realizado mediciones de BS de alcance nacional utilizando el módulo básico de Bienestar Autoreportado (BIARE), un instrumento que evalúa de manera global la felicidad, el balance entre emociones negativas y positivas, y la satisfacción con diferentes aspectos de la vida, incluida la satisfacción laboral. La evaluación que hacen las personas de su trabajo es muy importante, especialmente si se considera como un medio que permite satisfacer necesidades inmediatas y de socialización. Además, gran parte del bienestar social, material y de la propia vida están profundamente relacionados con las organizaciones y su dinámica (Calderón, Murillo, \& Torres, 2003; González, López-Guzmán, \& Sánchez, 2014; Ochoa, Jacobo, \& Leyva, 2012).

Aunque no existe un consenso respecto a la definición de bienestar en el trabajo (Peiró, Ayala, Tordera, Lorente, \& Rodríguez, 2014), se reconoce que contar con un trabajo interesante, que ofrece recompensas justas, condiciones laborales adecuadas y buenas relaciones con los compañeros de trabajo, favorece una alta satisfacción en el trabajo (Berg, 1999). La satisfacción con el trabajo depende tanto de elementos intrínsecos (e.g., compañeros de trabajo, la supervisión y el trabajo en sí) como extrínsecos (e.g., pago y promociones) (Diego, Diego, \& Olivar, 2001; Judge \& Klinger, 2008).

Así mismo, la participación de los empleados en el desarrollo de la organización, así como las actitudes positivas que presentan hacia el trabajo, están en función de la percepción que tengan del mismo (Juárez-Adauta, 2012; Luceño, Martín, Jaén, \& 
Pág 129

Dado que uno de los aspectos que impactan el Bienestar Subjetivo es la satisfacción con las condiciones de trabajo, niveles bajos de BS pueden impactar negativamente el nivel del compromiso del individuo con su organización.
Díaz, 2006; Mañas, Salvador, Boada, González, \& Agulló, 2007). Estas percepciones de la organización y el trabajo también tienen efectos importantes sobre la calidad de vida de los trabajadores e impactan su desempeño y logro de metas (Álvarez \& Muñiz, 2013; Moreno-Jiménez \& Gálvez, 2013; Valenzuela, Reygadas, \& Cruces, 2015; Werther, 2000). Además, cuando los empleados perciben baja satisfacción en el trabajo, su estabilidad emocional puede afectarse significativamente, derivando en altos índices de estrés, ansiedad o agotamiento emocional (Figueiredo-Ferraz, Grau-Alberola, Gil-Monte, \& García-Juesas, 2012; Luceño et al., 2006; Moreno-Jiménez \& Gálvez, 2013; Siegrist, 2005).

Al experimentar un menor bienestar en el trabajo, las contribuciones de los trabajadores en el desarrollo organizacional se ven mermadas, provocando toma de decisiones equivocadas y una mayor propensión al ausentismo (Dávila \& Jiménez, 2014; Lu, While, \& Barriball, 2005; Luceño et al., 2006; Sears, Shi, Coberley, \& Pope, 2013). Resulta importante que el trabajo tenga las características apropiadas para que la satisfacción de sus integrantes y su compromiso se mantengan, evitando disminuciones en su rendimiento, en la valoración de su trabajo y reduciendo así la rotación de personal (Álvarez \& Muñiz, 2013; Carreño, Medina-Mora, Martínez, Juárez, \& Vázquez. 2006; Pouliakas \& Theodossiou, 2010; Sears et al., 2013).

Dado que uno de los aspectos que impactan el BS es la satisfacción con las condiciones de trabajo, niveles bajos de BS pueden impactar negativamente el nivel del compromiso del individuo con su organización. Este compromiso, conocido como compromiso organizacional (CO), se ha definido como una actitud o estado de ánimo que deriva en comportamientos congruentes hacia la organización. Se refiere a la implicación e identificación que muestran las personas con la organización en la que laboran (Mowday, Steers, \& Porter, 1979).

Una persona podría sentirse comprometida con la organización por el deseo de permanecer en ella, por la obligación que representa el trabajo que desarrolla o por la necesidad de contar con un trabajo, sin que estos motivos sean excluyentes entre sí (Barraza \& Acosta, 2008; Frutos, Ruiz, \& San Martín, 1998; Meyer \& Allen, 1991).

Dentro de los componentes que integran el CO se encuentran tres factores: El factor afectivo hace referencia a los lazos emocionales que las personas forjan con la organización, al deseo de permanecer en ella y la satisfacción por estar ahí; esto refleja un apego emocional al percibir cubiertas sus expectativas respecto a su trabajo y la organización (Allen \& Meyer, 1990; Barraza \& Acosta, 2008; Blau, 2003; Frutos et al., 1998; Meyer \& Herscovitch, 2001; Meyer, Stanley, Herscovitch, \& Topolnytsky, 2002). El factor moral hace referencia al deseo de ser leal a la organización porque valoran su trabajo y la oportunidad de trabajar (Allen \& Meyer, 1996; Betanzos, Andrade, \& Paz, 2006; Meyer \& Allen, 1991). El tercer factor está constituido por la identificación de los trabajadores con los objetivos y valores institucionales. En la medida en que los empleados cuenten con valores y objetivos personales afines a su organización buscarán conseguir un desarrollo mutuo (Betanzos et al., 2006; Fonseca, Cruz, \& Chacón, 2019).

Estudios previos muestran que el CO se encuentra en relación positiva con la satisfacción con el trabajo y la productividad de las personas (Gómez-Sánchez, Recio, \& Avalos, 2012; Mañas et al., 2007; Moreno-Jiménez \& Gálvez, 2013). Asimismo, cuando las personas no se comprometen con el lugar de trabajo manifiestan comportamientos negativos como ausentismo, limitado o bajo logro en las metas planteadas, 
Pág 130

Algunos autores reportan una relación tan estrecha entre el Compromiso Organizacional y el Bienestar Subjetivo, que incluso los consideran consecuentes uno del otro. Dávila y Jiménez (2014) reportan que el Compromiso Organizacional, en conjunto con el sentido de pertenencia, permite predecir el Bienestar Subjetivo. Por su parte, otros investigadores encontraron en una muestra de trabajadores de la administración pública que el Compromiso Organizacional está influenciado en gran medida por la satisfacción laboral, la satisfacción por la vida y el bienestar psicológico (Mañas et al., 2007). y abandono del empleo (Lu et al., 2005; Luchak \& Gellatly, 2007). En correspondencia, los empleados confían y se comprometen con su organización en la medida en que perciban que la organización a su vez está comprometida con ellos y les brinda apoyo (Soltani \& Ali, 2016).

Algunos autores reportan una relación tan estrecha entre el CO y el BS, que incluso los consideran consecuentes uno del otro. Dávila y Jiménez (2014) reportan que el $\mathrm{CO}$, en conjunto con el sentido de pertenencia, permite predecir el BS. Por su parte, otros investigadores encontraron en una muestra de trabajadores de la administración pública que el CO está influenciado en gran medida por la satisfacción laboral, la satisfacción por la vida y el bienestar psicológico (Mañas et al., 2007). Estos resultados apuntan hacia dos posibilidades distintas: por una parte, que el CO predice el BS y, por otra, lo contrario, es el BS el que deriva en mayor CO. A estas posibilidades consideramos necesario sumar una tercera hipótesis de una relación recíproca y positiva entre ambas variables.

Dada la necesidad de aportar al conocimiento de la relación entre el BS y el CO, el objetivo del presente estudio fue especificar la dirección de esta relación, analizando para ello las variables en una muestra de trabajadores de una organización educativa pública en México. Utilizando análisis de modelos de ecuaciones estructurales, se contrastaron las hipótesis de relaciones en un solo sentido con una hipótesis de modelo no recursivo que plantea la posibilidad de retroalimentación entre ambos constructos. Además, dado que no se cuenta con antecedentes de las propiedades psicométricas del BIARE (INEGI, 2016) en términos de confiabilidad y validez, se establece como objetivo secundario analizar estas propiedades.

\section{Método}

\section{Participantes}

Los participantes en el estudio fueron 230 empleados de una de las principales universidades públicas de México, la cual cuenta con más de 80 años de historia y sedes en diferentes regiones del país. De los 230 empleados, 180 forman parte de la sede de Guanajuato y 50 laboran en la Ciudad de México. El 49\% de los participantes son hombres y el $51 \%$ mujeres. Al momento de la aplicación el $6.3 \%$ reportó contar con menos de un año de antigüedad en la Universidad, 59.2\% dijeron tener entre uno y cinco años, $18.4 \%$ reportaron tener entre seis y 10 años de antigüedad y el $16.1 \%$ restante contaba con más de 11 años. De acuerdo con la actividad laboral, 133 era personal docente y 90 personal administrativo. Respecto al estatus de su contrato, el $79.5 \%$ de los participantes contaban con una plaza en propiedad o de base, y el $20.5 \%$ tienen un contrato de interinato. Y en relación con algunos datos sociodemográficos, el $41.1 \%$ no contaba con una pareja, el 58.9\% si; y el 54.9\% reportó tener por lo menos un hijo.

\section{Instrumentos}

Instrumento de Compromiso Organizacional

Desarrollado y validado por Fonseca, Cruz y Chacón (2019), mide el CO identificando los factores afectivo (e.g. Disfruto mucho trabajar en esta organización), moral (e.g. Esta institución merece mi lealtad) y de identificación con objetivos y valores (e.g. Me identifico con los objetivos de esta institución). Estas subescalas han obtenido valores de alfa de Cronbach de $.89, .87$ y .86 respectivamente, y la estructura general del instrumento fue verificada previamente mediante análisis factoriales exploratorios y confirmatorios (Fonseca, Cruz, \& Chacón, 2019). Cada factor cuenta con cuatro 
reactivos, presentados en un formato de respuesta tipo Likert, utilizando una escala gráfica de cinco cuadrados que representan un continuo del más pequeño al más grande, siendo los valores "totalmente en desacuerdo", para el cuadrado más pequeño, y "totalmente de acuerdo", para el cuadrado más grande. Aunque el principal referente de medición del CO es el instrumento de Allen y Meyer (1990), este fue diseñado considerando la población canadiense. Aunque ha sido traducido y adaptado a otros contextos (Arias, 2001; Barraza \& Acosta, 2008), para el presente proyecto se prefirió optar por un instrumento desarrollado desde el contexto cultural mexicano.

\section{Módulo básico del Bienestar Autorreportado (BIARE)}

Desarrollado por el INEGI (2016), para evaluar el BS. Este instrumento se ha utilizado para mediciones a nivel nacional en México y contiene entre los reactivos que lo integran una evaluación de satisfacción en el trabajo. Se eligió este instrumento para medir el BS porque ha sido utilizado en mediciones de índole nacional, pero al mismo tiempo se desconocen evidencias de su validez y confiabilidad, constituyendo un área de oportunidad que puede resolverse inicialmente en el presente proyecto. Retomando los fundamentos planteados por Diener (1984), este instrumento hace referencia a las percepciones sobre la situación y el contexto que tienen los individuos en edad adulta (INEGI, 2016). Abarca tres aspectos: la satisfacción con la vida, el balance afectivo y la felicidad. La satisfacción con la vida se califica con valores de 0 a 10 , se realiza primero la pregunta de manera global (En general, ¿Qué tan satisfecho/a se encuentra con su vida?), posteriormente se realiza la misma pregunta enfocada a dominios específicos (i.e. tiempo libre, logros personales, situación económica, trabajo, vivienda, vida familiar, afectiva y social). El balance afectivo surge de la sumatoria de la evaluación en una escala de 0 a 10 de estados de ánimo positivos (alegría y tranquilidad) y negativos (enojo y tristeza), experimentados el día anterior al diligenciamiento del cuestionario. La felicidad cuenta con un reactivo que es evaluado en una escala de 0 a 10, que hace referencia de manera general a la vida en su conjunto. Dado que en esos reportes no se presentan análisis que permitan verificar la validez y confiabilidad de este instrumento, estas propiedades serán verificadas en el presente estudio.

\section{Procedimiento}

En primera instancia, se obtuvo la autorización pertinente de los directivos de la universidad para realizar el estudio; luego se realizó la convocatoria evitando el uso de los medios internos de comunicación y de los logos institucionales de acuerdo con lo solicitado por los directivos. Siguiendo los lineamientos del código ético del Psicólogo (Sociedad Mexicana de Psicología, 2007) se informó a los interesados sobre los objetivos de investigación del proyecto, y que su participación era voluntaria, que no habría recompensas por participar ni sanciones por no hacerlo; incluso, que si decidían participar podían abandonar el estudio o dejar preguntas sin responder si así lo deseaban. Así mismo, se les informó que sus datos serían totalmente anónimos, dado que no se solicitaba su nombre u otros datos personales que permitieran su identificación personal; y, además, confidenciales, es decir, quedaban a resguardo de los investigadores, sin que se facilitaran de ninguna manera a las autoridades de su institución y serían analizados solo con fines de investigación científica. A quienes aceptaron participar, se les solicitó la firma del respectivo consentimiento informado, se les entregó el cuadernillo con los instrumentos y se les asesoró para diligenciarlo. La mayoría de las aplicaciones se realizaron de forma individual, aunque algunos se aplicaron grupalmente de acuerdo con la disponibilidad del personal y sus cargas de trabajo. 
Pág 132

\section{Resultados}

Con el fin de obtener evidencias de la validez del constructo BS evaluado a través del BIARE, se realizó un análisis factorial exploratorio de máxima verosimilitud con rotación ortogonal varimax de la dimensión de satisfacción con la vida, utilizando para ello el paquete de análisis estadísticos SPSS en su versión 22 (IBM, 2013), teniendo como resultado una estructura de tres factores o componentes de satisfacción. El primero, satisfacción general, se compone de 6 reactivos (i.e. En general, ¿Qué tan satisfecho/a se encuentra con su vida?, ¿Qué tan satisfecho se siente con? El tiempo libre, sus logros personales, su situación económica, su trabajo) que explican el $18.81 \%$ de la varianza total y obtiene un indicador de consistencia interna $\mathrm{a}=.771$. El segundo componente fue nombrado satisfacción social-afectiva, el cual cuenta con tres reactivos (e.g. ¿Qué tan satisfecho se siente con?, su vida afectiva, su vida social, su vida familiar) que explican el $15.22 \%$ de la varianza total, mostrando un índice de consistencia interna $\alpha=.801$. El tercer componente fue nombrado satisfacción nivel de vida, el cual cuenta con tres reactivos (e.g. ¿Qué tan satisfecho se siente con?, su vecindario o colonia, su vivienda, su educación) que explican el $13.12 \%$ de la varianza total con un índice de consistencia interna $\alpha=.679$.

A fin de poner a prueba la idea del BS como un constructo multidimensional, se realizó un análisis factorial confirmatorio mediante ecuaciones estructurales, utilizando el programa IBM SPSS AMOS 22 (Arbuckle, 2013). El modelo, que se muestra en la $\underline{\mathrm{Fi}-}$ gura 1, incluye las tres dimensiones identificadas en el análisis factorial exploratorio de satisfacción con la vida (i.e. satisfacción general, satisfacción social-afectiva y satisfacción nivel de vida), más las dimensiones de balance afectivo y felicidad. Los resultados del análisis no muestran discrepancias estadísticamente significativas entre el modelo propuesto teóricamente y los datos analizados $\left(\mathrm{Ch}^{2}=4.819 ; \mathrm{gl}=4\right.$; $p=0.306$ ). El indicador RMR presentó un valor igual a .022, lo cual indica que existe poca varianza restante después de la explicada por el modelo teórico. El valor para el indicador RMSEA fue de .031 [IC $90 \%=.00, .11$ ]. El GFI fue igual a .991 y el CFI igual a .997. Estos indicadores en conjunto reflejan un ajuste adecuado entre los datos y el modelo teórico (Brown, 2006).

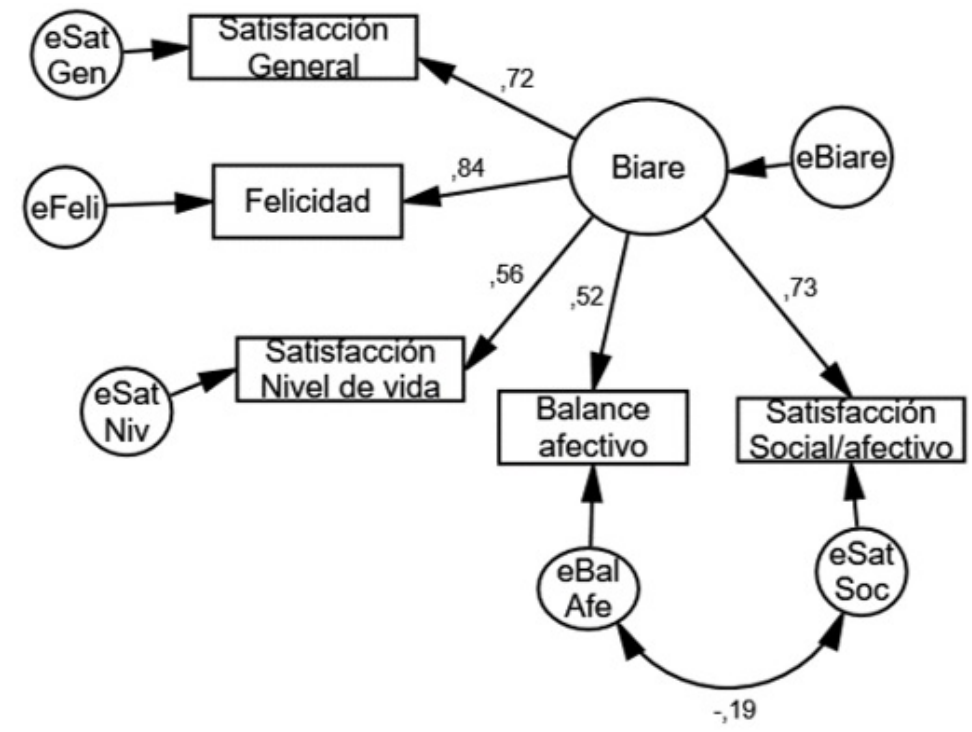

Figura 1. Análisis Factorial Confirmatorio para la medición de BIARE. Los coeficientes de las vías corresponden a coeficientes estandarizados 
El instrumento para evaluar el CO mostró buenos indicadores de consistencia interna y bondad de ajuste en análisis factoriales exploratorios y confirmatorios, reflejando una estructura de tres factores que son: afectivo $(\boldsymbol{a}=.820)$, identificación con objetivos y valores $(\boldsymbol{\alpha}=.803)$ y moral $(\boldsymbol{\alpha}=.825)$.

Ya verificados los indicadores de validez y confiabilidad de ambos instrumentos, se llevó a cabo un primer modelo de ecuaciones estructurales mediante el software AMOS (Arbuckle, 2013) estableciendo efectos del BIARE hacia el CO. El indicador Chi muestra diferencias estadísticamente significativas entre el modelo propuesto y las covarianzas presentes en los datos $\left(\mathrm{Chi}^{2}=65.627\right.$; $\left.\mathrm{gl}=18 ; \mathrm{p}<.001\right)$. El indicador $R M R$ presentó un valor igual a .074, mientras que el valor para la RMSEA fue de .111 [IC $90 \%=.08, .14 \mathrm{l}$. El GFI fue igual a .931 y el CFI igual a .915. Estos indicadores reflejan un ajuste general inadecuado entre los datos y el modelo teórico (Brown, 2006) y serán contrastados con los modelos siguientes. Como se esperaba, los efectos del BIARE sobre el CO son positivos, es decir, cada vez que el bienestar se incrementa en una desviación estándar, el CO se incrementa en .36 desviaciones estándar (C.R. =4.70, $p<.001)$. Cuando las personas se sienten satisfechas con su vida en general, tienden a reportarse también más comprometidas con su organización. Sin embargo, el sentido de esta relación (BIARE $\rightarrow \mathrm{CO}$ ) está determinada por la forma en que fue solicitada en el análisis; si se pide en el sentido inverso (CO $\rightarrow$ BIARE) el efecto es también positivo y de la misma magnitud, pero en este caso del CO hacia el BIARE. Para establecer en detalle la naturaleza de las relaciones entre estos dos constructos y analizar un posible efecto de retroalimentación entre ellos es necesario analizar simultáneamente ambas relaciones, convirtiendo el modelo de ecuaciones estructurales en un modelo no recursivo (Kline, 2011).

Este modelo no recursivo, que analiza los efectos recíprocos entre el BIARE y el CO es idéntico al obtenido en el análisis previo donde los efectos van del BIARE al CO, con excepción de la varianza entre estos dos constructos que ahora se muestra desglosada. Como se observa en la Figura 2, los efectos que van del BIARE al CO son el doble de los que van del CO hacia el BIARE.

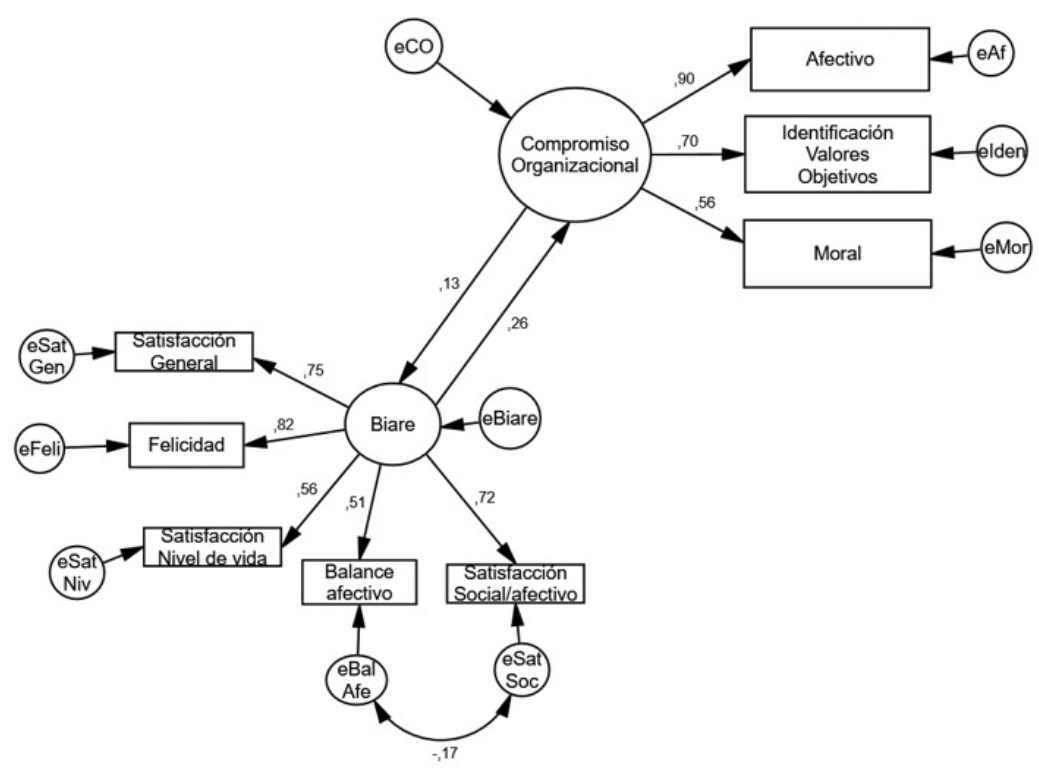

Figura 2. Modelo de Ecuaciones Estructurales no recursivo, analizando relaciones recíprocas entre el BIARE y el CO. Se muestran valores estandarizados. 
Sin embargo, los pobres indicadores de ajuste previamente reportados y la revisión de las discrepancias en las relaciones entre variables sugieren que algunos componentes del BIARE y del CO guardan relaciones entre sí adicionales a las que se observan entre los constructos de forma global. Estas relaciones fueron analizadas y se presentan en la Figura 3. En particular, se observa que el efecto del CO hacia el BIARE se debe principalmente a un efecto positivo sobre el factor de satisfacción general (C.R. $=5.375, p<.001)$, haciendo que el efecto previamente identificado del CO sobre el BIARE pierda peso y significancia estadística. Además, se observa un efecto directo y positivo del componente satisfacción social-afectiva del BIARE sobre el factor moral del CO (C.R. =3.612. $p<.001)$. Esta relación es adicional al efecto del BIARE hacia el CO, la cual sigue siendo estadísticamente significativa ( $C . R .=3.209 . p=.001)$. Este modelo presenta mejores indicadores de bondad de ajuste que los dos anteriores, ubicándose ya dentro de parámetros aceptables para el indicador $\mathrm{Chi}^{2}\left(\mathrm{Chi}^{2}=21.16 ; \mathrm{gl}=16\right.$; $p=.172)$ y para todos los demás indicadores ( $R M R=.043 ; G F I=.976 ; C F I=.991 ; R M S E A$ $=.039$ [IC 90\% =.000, .079]) (Kline, 2011).

Este modelo muestra que altos niveles de CO se asocian a una mejor evaluación de satisfacción con la vida en general, pero no con las otraos dimensiones particulares, como la felicidad, el balance afectivo, la satisfacción social-afectiva o nivel de vida, las cuales deberían responder a otras esferas de la vida (e.g., vida familiar, vivienda, educación). Por su parte, los efectos del BIARE sobre el CO se presentan tanto de manera global, como a través de los efectos de la satisfacción social-afectiva, que presentan un efecto positivo hacia el componente moral del CO.

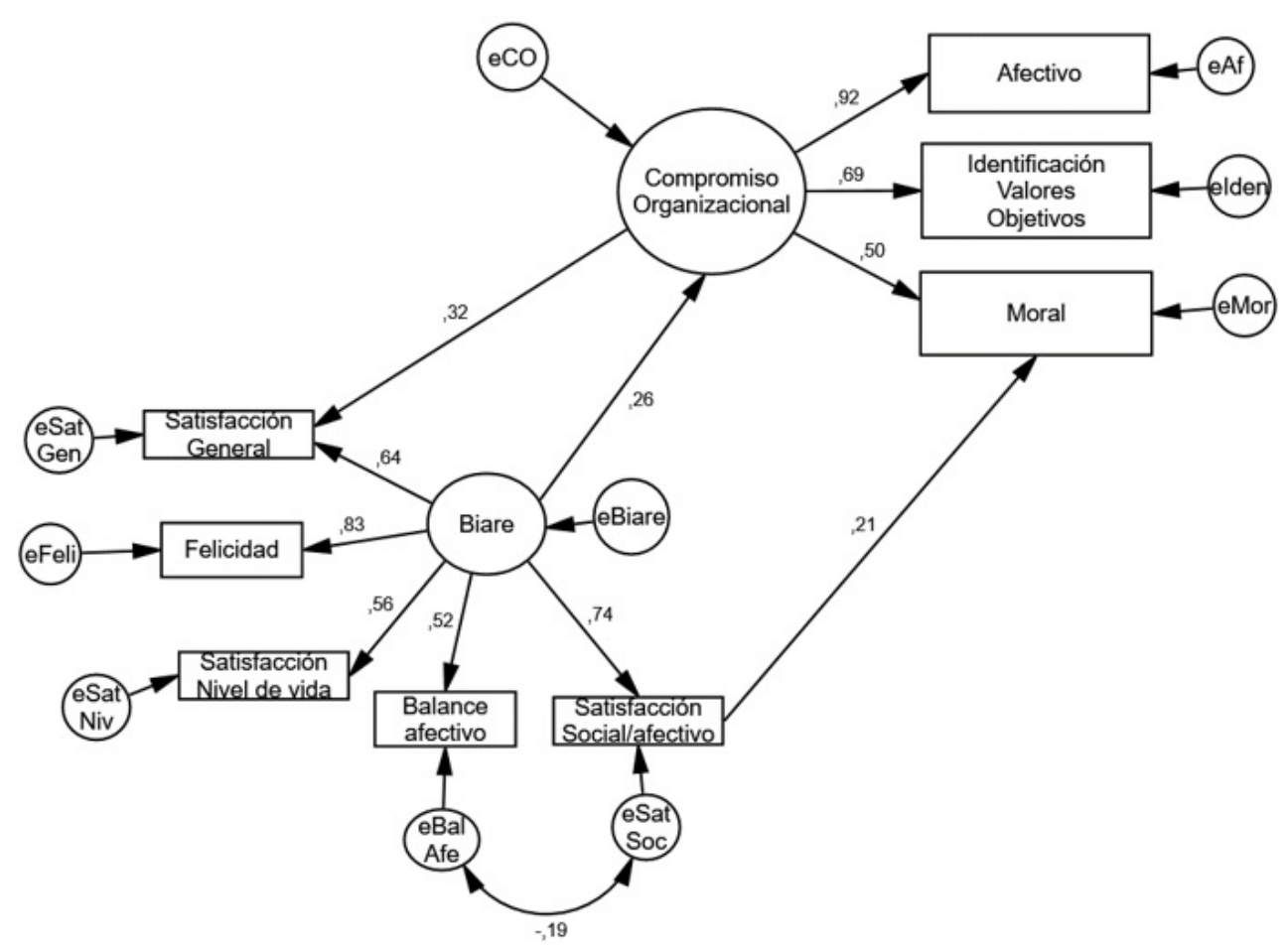

Figura 3. Modelo de Ecuaciones Estructurales no recursivo, analizando efectos directos entre los componentes del CO y el BIARE. Se muestran valores estandarizados. 
El factor de compromiso moral en particular ha sido previamente identificado como un facilitador para la aceptación del cambio organizacional en estudios previos (Yousef, 2016), por lo que analizar el tipo de vínculos sociales que tienden a establecer los individuos puede ser un criterio valioso para los procesos de selección, en particular en organizaciones que requieren una alta tolerancia a los cambios organizacionales de sus colaboradores.

\section{Discusión y conclusiones}

Para alcanzar el objetivo de contrastar la hipótesis de relación entre los constructos BS y CO, se llevó a cabo un análisis de modelos de ecuaciones estructurales, en el cual el primer modelo establecía una relación en un solo sentido, y el segundo y tercero planteaban la posibilidad de retroalimentación entre ambos constructos. En la muestra evaluada, se encontró una relación positiva en un sentido que indica que el BS precede al CO, lo cual tiene soporte en los hallazgos enunciados por Mañas et al.

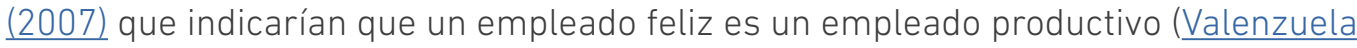
et al., 2015). En el sentido opuesto, también se encontraron efectos significativos y positivos del CO en el BS, indicando que el beneficio de un alto BS entre los empleados de cualquier organización aportará significativamente tanto en el crecimiento de la persona (Álvarez \& Muñiz, 2013; Moreno-Jiménez \& Gálvez, 2013; Valenzuela et al., 2015) como en el de la propia organización (Dávila \& Jiménez, 2014; Luceño et al., 2006; Sears et al., 2013), permitiendo el establecimiento de puntos de equilibrio basados en estas relaciones recíprocas de ganar-ganar entre ambas partes.

Los efectos observados y no esperados entre el componente satisfacción social/ afectiva del BIARE y el factor moral del CO podrían deberse a una diferencia individual en los participantes: personas que establecen vínculos afectivos estables y duraderos con los demás lo hacen también con su institución, por lo cual asumen un compromiso moral, de deuda y gratitud con los círculos sociales a los cuales sienten pertenecer (e.g., familia, amigos) incluido los de su empleo. Es decir, personas que acostumbran a mantener relaciones sociales de largo plazo en las cuales muestran mucho compromiso podrían ser también personas más comprometidas moralmente con su organización. El factor de compromiso moral en particular ha sido previamente identificado como un facilitador para la aceptación del cambio organizacional en estudios previos (Yousef, 2016), por lo que analizar el tipo de vínculos sociales que tienden a establecer los individuos puede ser un criterio valioso para los procesos de selección, en particular en organizaciones que requieren una alta tolerancia a los cambios organizacionales de sus colaboradores.

Resulta importante prestar atención al BS de las personas en sus trabajos dado su efecto en cuestiones de productividad y crecimiento de las organizaciones. Al sentirse bien con la organización para la que colaboran las personas se comprometerán más con ésta, beneficiándola significativamente. Además, dados los impactos del $\mathrm{CO}$ en el BS de los trabajadores, es necesario que las organizaciones reconozcan como parte de su responsabilidad social cuidar y propiciar un clima que favorezca la reciprocidad y compromiso moral, el afecto positivo hacia la organización y la identificación de sus colaboradores con los principios y valores organizacionales, componentes estos del CO. Estudios como el de Matthews, Mills, Trout y English (2014) muestran que las acciones de apoyo que las organizaciones brindan a los trabajadores son entendidas por estos últimos como señales de compromiso, las cuales impactan positivamente en su BS. Las gestiones que generan alto CO de sus colaboradores impactan positivamente en su BS, y un mal ambiente laboral impactaría negativamente su BS, obteniendo a su vez efectos negativos sobre su CO y en esa medida sobre el funcionamiento de su organización. Las opciones, dada la relación recíproca entre $\mathrm{BS}$ y $\mathrm{CO}$, son entonces alimentar un círculo virtuoso de crecimiento y bienestar mutuo, o bien descuidar la relación entre la organización y sus colaboradores, generando un círculo vicioso que afecta negativamente a ambas partes con insatisfacción, baja productividad y alta rotación de personal. Consideramos también necesario hacer una anotación respecto a delimitar el $\mathrm{CO}$ a fin de no confundirlo o que no derive en un problema adicción al trabajo, legitimado bajo la 
etiqueta de compromiso con el trabajo (Shimazu, Schaufeli, Kamiyama, \& Kawakami, 2015), que podría derivar en efectos negativos para ambas partes como ausentismos por problemas de salud y un desgaste que derivaría en insatisfacción y falta de compromiso hacia la organización. Debe considerarse que las horas y energía que se destinan al trabajo entran en conflicto con el tiempo y energía requeridos para otras actividades como las familiares, generando problemas que requieren un proceso de ajuste del trabajador y su familia para no afectar su BS (Matthews, Wayne \& Ford, 2014).

La presente investigación aporta elementos para considerar al BS y al CO como factores que se influencian entre sí y que a su vez influyen en el desarrollo de cualquier organización. Surge así la necesidad de contrastar los resultados con los de otros sectores, por ejemplo, el de la educación privada u otros ramos de la administración pública y privada, a fin de fortalecer los hallazgos que apuntan a esperar que una persona con alto BS será una persona altamente comprometida con la institución y viceversa. Consideramos que la muestra de la presente investigación, integrada tanto de profesores como de personal administrativo, es valiosa para los fines de análisis de una organización, sin embargo, debe reconocerse la dificultad de comparar estos resultados con otros estudios que analizan muestras solo de personal docente, por ejemplo, el de González, Torres y Carreres (2015), dado que la naturaleza de las labores y condiciones de trabajo del personal administrativo son muy diferentes. También resultaría valioso analizar con mediciones más precisas los efectos del CO sobre cada uno de los componentes de la satisfacción, en particular con la satisfacción laboral, constructo que se evalúa en esta investigación solo como un elemento del BS y no de manera independiente.

El trabajo es una actividad que permite a las personas satisfacer distintas necesidades en el plano económico y de supervivencia, permitiendo su desarrollo personal. Cuando las personas disfrutan de su actividad laboral diaria, les genera una sensación de plenitud y productividad en función de la percepción que tienen del trabajo que realizan y de la satisfacción que les produce involucrarse con la organización (Valenzuela et al., 2015). Dicha satisfacción en el trabajo contribuye tanto al bienestar general de las personas como al compromiso que manifiestan hacia la organización en la que colaboran. Los resultados de esta investigación ofrecen mayor precisión al ofrecer evidencias de relaciones de retroalimentación entre los componentes y factores del BS y el CO.

\section{Referencias}

Abello-Llanos, R., Amaris-Macías, M., Blanco-Abarca, A., Madariaga-Orozco, C., Manrique-Palacio, K., Martínez-González, M., ... Díaz-Méndez, D. (2009). Bienestar y trauma en personas adultas desplazadas por la violencia política, Universitas Psychologica, 8(2), 455-470. Recuperado de https://revistas.javeriana.edu.co/ index.php/revPsycho/article/view/489

Allen, N., \& Meyer, J. (1990). The measurement and antecedents of affective, continuance and normative commitment to the organization, Journal of Occupational Psychology, 63, 1-18. doi: https://doi.org/10.1111/j.2044-8325.1990.tb00506.x

Allen, N., \& Meyer, J. (1996). Affective, Continuance, and Normative Commitment to the Organization: An Examination of Construct Validity, Journal of Vocational Behavior, 49, 252-276. doi: https://doi.org/10.1006/jvbe.1996.0043 
Álvarez, A., \& Muñiz, J. (2013). Felicidad y desarrollo de la Cultura en las organizaciones, un enfoque psicosocial, Revista de comunicación, 12, 7-31. http://web.a.ebscohost.com/ehost/detail/detail?vid=0\&sid=11a7268a-9796-4ba4-9ef8-05a3c6

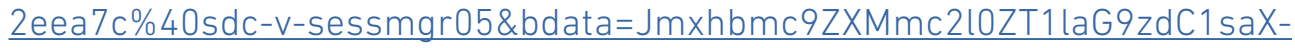
Zl\#db=a9h\&AN=92630498

Arbuckle, J. L. (2013). Amos (Versión 22.0) [Programa de cómputo]. Chicago: SPSS.

Arias, F. (2001). El compromiso personal hacia la organización y la intención de permanencia: algunos factores para su incremento, Revista Contaduría y Administración, 200, 5-12. https://s3.amazonaws.com/academia.edu. documents/30255878/4509-4441-0-PB.pdf?AWSAccessKeyld=AKIAIWOWYYGZ2Y53UL3A\&Expires $=1554400729 \&$ Signature $=$ RalQCPcwd120YUFBBVplxwpeJuw\%3D\&response-content-disposition=inline \%3B\%20filename\%3DEl compromiso personal hacia_la_organiza.pdf

Barraza, A., \& Acosta, M. (2008). Compromiso organizacional de los docentes de una institución de educación media superior, Innovación Educativa, 8(45), 20-35. https://www.redalyc.org/html/1794/179420818003/

Berg, P. (1999). The Effects of High Performance Work Practices on Job Satisfaction in the United States Steel Industry, Relations Industrielles/ Industrial Relations, 54(1), 111-135. doi: https://doi.org/10.7202/051222ar

Betanzos, N., Andrade, P., \& Paz, F. (2006). Compromiso organizacional en una muestra de trabajadores mexicanos, Revista de Psicología del Trabajo y de las Organizaciones, 22(1), 25-43. https://www.redalyc.org/html/2313/231317045002/

Blau, G. (2003). Testing for a four-dimensional structure of occupational commitment, Journal of Occupational and Organizational Psychology, 76, 469-488. doi: https://doi. org/10.1348/096317903322591596

Bowling, N. A., Eschleman, K. J., \& Wang, Q. (2010). A meta-analytic examination of the relationship between job satisfaction and subjective well-being. Journal of Occupational and Organizational Psychology, 83(4), 915-934. doi: https://doi. org/10.1348/096317909X478557

Brown, T. (2006). Confirmatory Factor Analysis for Applied Research, EUA: The Guilford Press.

Calderón, G., Murillo, S., \& Torres, K. (2003). Cultura organizacional y bienestar laboral, Cuadernos de Administración, 16(25), 109-137. https://revistas.javeriana.edu.co/ index.php/cuadernos admon/article/view/5452

Carr, D., Freedman, V. A., Cornman, J. C., \& Schwarz, N. (2014). Happy marriage, happy life? Marital quality and subjective well-being in later life. Journal of Marriage and Family, 76(5), 930-948. https://doi.org/10.1111/jomf.12133

Carreño, S., Medina-Mora, M.E., Martínez, N., Juárez, F., \& Vázquez, L. (2006). Características organizacionales, estrés y consumo de alcohol en trabajadores de una empresa textil Mexicana, Salud Mental, 29(4), 63-70. http://www.scielo.org.mx/ scielo.php?script=sci arttext\&pid=S0185-33252006000400063

Dávila, C., \& Jiménez, G. (2014). Sentido de pertenencia y compromiso organizacional: predicción del bienestar, Revista de Psicología, 32(2), 271-302. http://www. scielo.org.pe/scielo.php?pid=S0254-92472014000200004\&script=sci arttext

Díaz, D., Rodríguez-Carvajal, R., Blanco, A., Moreno-Jiménez, B., Gallardo, I., Valle, C., \& Dierendonck, D. (2006). Adaptación española de las escalas de bienestar psicológico de Ryff, Psicothema, 18(3), 572-577.

Diego, R., Diego, J., \& Olivar, S. (2001). Job Satisfaction in Banking Workers, Psicothema, 13(4), 629-635. Recuperado de http://www.psicothema.com/pdf/490.pdf

Diener, E. (1984). Subjective Well-Being, Psychological Bulletin, 95 (3), 542-575. doi: http://dx.doi.org/10.1037/0033-2909.95.3.542 
Diener, E. (2000). Subjective Well-Being the Science of Happiness and a Proposal for a National Index, American Psychologist, 55(1), 34.43. doi: https://doi. org/10:1037//0003-066X.55.1.34

Diener, E., Oishi, S., \& Lucas, R. (2003). Personality, culture and subjective well-being: Emotional and cognitive evaluations of life. Annual Review of Psychology, 54, 403425. doi: https://doi.org/10.1146/annurev.psych.54.101601.145056

Diener, E., Oishi, S., \& Lucas, R. E. (2015). National accounts of subjective well-being. American Psychologist, 70(3), 234-242. doi: http://dx.doi.org/10.1037/ a0038899

Figueiredo-Ferraz, H., Grau-Alberola, E., Gil-Monte, P.R., \& García-Juesas, J.A. (2012). Síndrome de quemarse por el trabajo y satisfacción laboral en profesionales de enfermería, Psicothema, 24(2), 271-276.

Fonseca, J., Cruz, C., \& Chacón, L. (2019). Validación del instrumento de compromiso organizacional en México: Evidencias de validez de constructo, criterio y confiabilidad. Revista de Psicología, 37(1), 1-22. doi: https://doi.org/10.18800/ psico.201901.001

Frutos de, B., Ruiz, M., \& San Martin, R. (1998). Análisis factorial confirmatorio de las dimensiones del compromiso con la organización, Psicológica, 19, 345-366.

Garaigordobil, M., Aliri, J., \& Fontaneda, I. (2009). Bienestar psicológico subjetivo: Diferencias de sexo, relaciones con dimensiones de personalidad y variables predictoras, Behavioral Psychology/Psicología Conductual, 17(3), 543-559.

Galinha, I. C., Garcia-Martin, M. Á., Oishi, S., Wirtz, D., \& Esteves, F. (2016). Cross-cultural comparison of personality traits, attachment security, and satisfaction with relationships as predictors of subjective well-being in India, Sweden, and the United States. Journal of Cross-Cultural Psychology, 47(8), 1033-1052.

García, M. (2002). El bienestar subjetivo, Escritos de Psicología, 6, 18-39.

Gómez-Sánchez, D., Recio, R., \& Avalos, M. (2012). Compromiso organizacional y su relación con la satisfacción laboral de los empleados en RIOVERDE, SLP. Trabajo presentado en Retos y alcances de la Investigación Conductual 1er Congreso Nacional de Ciencias del Comportamiento. San Luis Potosí, México. Resumen recuperado de http://www.eumed.net/libros-gratis/2013a/1336/empleados-rioverde.html

González, F., López-Guzmán, T., \& Sánchez, S. (2014). Satisfacción laboral y compromiso organizativo en los recursos humanos de la hostelería de Córdoba (España): Influencia de la tipología contractual y la jornada de trabajo, Intangible Capital, 10(1), 189-211. http://hdl.handle.net/2099/14479

González, T. M. P., Torres, L. H., \& Carreres, A. L. (2015). Felicidad subjetiva y autoeficacia docente en profesorado de República Dominicana y España. European Journal of Investigation in Health, Psychology and Education, 3(3), 277-288. doi: https://doi.org/10.30552/ejihpe.v3i3.55

IBM Corp. (2013). IBM SPSS Statistics for Windows, Versión 22.0. Armonk, NY: IBM Corp.

Instituto Nacional de Estadística Geografía e Información (INEGI). (2016). Indicadores de Bienestar Autorreportado de la Población Urbana (Boletín de prensa 116/16). México.

Juárez-Adauta, S. (2012). Clima organizacional y satisfacción laboral. Revista médica del instituto Mexicano del Seguro Social, 50(3), 307-314. https://www.redalyc.org/ html/4577/457745495014/

Judge, T., \& Klinger, R. (2008). Job Satisfaction Subjective Well-Being at Work. En Eid M. \& Larsen, R. (Eds.), The Science of Subjective Well-Being (pp. 393-413). New York: The Guilford Press. 
Kline, R. (2011). Principles and practice of structural equation modelling. New York: The Guilford Press.

Larsen, R., \& Eid, M. (2008). Ed Diener and the Science of Subjective Well-Being. En Eid M. \& Larsen, R. (Eds.), The Science of Subjective Well-Being (pp. 1-13). New York: The Guilford Press.

Lu, H., While, A.E., \& Barriball, K.L. (2005). Job satisfaction among nurses: A literature review. International Journal of Nursing Studies, 42, 211-227. https://doi. org/10.1016/j.ijnurstu.2019.01.011

Luceño, L., Martín, J., Jaén, M., \& Díaz, E. (2006). Psycho-social risks and trait anxiety as predictors of stress and job satisfaction, Ansiedad y Estrés, 12(1), 89-97.

Luchack, A., \& Gellatly, I. (2007). A Comparison of Linear and Nonlinear Relations Between Organizational Commitment and Work Outcomes, Journal of Applied Psychology, 92(3), 786-793. doi: https://doi.org/10.1037/0021-9010.92.3.786

Mañas, M., Salvador, C., Boada, J., González, E., \& Agulló, E. (2007). La satisfacción y el bienestar psicológico como antecedentes del compromiso organizacional, Psicothema, 19(3), 395-400.

Marrero, R., Carballeira, M., \& González, J. (2014). Relación entre bienestar subjetivo, optimismo y variables sociodemográficas en estudiantes universitarios de la Universidad de San Luis Potosí en México, Universitas Psychologica, 13(3), 10831098. doi: https://doi.org/10.11144/Javeriana.UPSY13-3.rbso

Matthews, R. A., Mills, M. J., Trout, R. C., \& English, L. (2014). Family-supportive supervisor behaviors, work engagement, and subjective well-being: A contextually dependent mediated process. Journal of occupational health psychology, 19(2), 168. doi: https://doi.org/10.1037/a0036012

Matthews, R. A., Wayne, J. H., \& Ford, M. T. (2014). A work-family conflict/subjective well-being process model: A test of competing theories of longitudinal effects. Journal of Applied Psychology, 99(6), 1173. doi: http://dx.doi.org/10.1037/a0036674

Meyer, J., \& Allen, N. (1991). A three-component conceptualization of organizational commitment, Human Resource Management Review, 1(1), 61-89. doi: https:// doi.org/10.1016/1053-4822(91)90011-Zdoi:10.1016/1053-4822(91)90011-Z doi:10.1016/1053-4822(91)90011-Z

Meyer, J., \& Herscovitch, L. (2001). Commitment in the workplace toward a general model, Human Resource Management Review, 11, 299-326. doi: https://doi.org/10.1016/ s1053-4822(00)00053-x

Meyer, J., Stanley, D., Herscovitch, L., \& Topolnytsky, L. (2002). Affective, Continuance, and Normative Commitment to the Organization: A Meta-analysis of Antecedents, Correlates, and Consequences, Journal of Vocational Behavior, 61, 20-52. doi: https://doi.org/10.1006/jvbe.2001.1842

Moreno-Jiménez, B., \& Gálvez, M. (2013). El efecto del distanciamiento psicológico del trabajo en el bienestar y la satisfacción con la vida: un estudio longitudinal, Journal of Work and Organizational Psychology, 29, 145-151.

Mowday, R., Steers, R., \& Porter, L. (1979). The Measurement of Organizational Commitment, Journal of Vocation Behavior, 14, 224-247. doi: https://doi.org/10.1016/00018791(79)90072-1

Ochoa, S., Jacobo, C., \& Leyva, B. (2012). Agenda de investigación para el estudio de las organizaciones: un acercamiento multidisciplinario. En: Rodríguez, H., Caldera, D. y Ortega, M. (Eds). El aprendizaje colaborativo en el análisis organizacional Experiencias y avatares. PEARSON.

Okbay, A., Baselmans, B. M., De Neve, J. E., Turley, P., Nivard, M. G., Fontana, M. A., ... Gratten, J. (2016). Genetic variants associated with subjective well-being, depressive symptoms, and neuroticism identified through genome-wide analyses. Nature genetics, 48(6), 624. doi: https://doi.org/10.1038/ng.3552 
Peiró, J. M., Ayala, Y., Tordera, N., Lorente, L., \& Rodríguez, I. (2014). Bienestar sostenible en el trabajo: Revisión y reformulación. Papeles del psicólogo, 35(1), 5-14. https://www.redalyc.org/html/778/77830184002/

Pouliakas, K., \& Theodossiou, I. (2010). Diferencias de satisfacción con el trabajo en Europa según nivel salarial. Revista Internacional del Trabajo, 129(1), 1-32. doi: https://doi.org/10.1111/i.1564-9148.2010.00073.x

Rojas, M., \& Elizondo-Lara, M. (2012). Satisfacción de vida en Costa Rica un enfoque de dominios de vida, Latin American Research Review, 47(1), 78-94.

Sánchez-Álvarez, N., Extremera, N., \& Fernández-Berrocal, P. (2016). The relation between emotional intelligence and subjective well-being: A meta-analytic investigation. The Journal of Positive Psychology, 11(3), 276-285. doi: https://doi.org/10.1080/17439760. $\underline{2015.1058968}$

Sears, L., Shi, Y., Coberley, C., \& Pope, J. (2013). Overall Well-Being as a Predictor of Health Care, Productivity, and Retention Outcomes in a Large Employer. Population Health Management, 16 (6),397-405. doi: https://doi.org/10.1089/ pop.2012.0114

Siedlecki, K. L., Salthouse, T. A., Oishi, S., \& Jeswani, S. (2014). The relationship between social support and subjective well-being across age. Social indicators research, 117(2), 561-576. doi: https://doi.org/10.1007/s11205-013-0361-4

Shimazu, A., Schaufeli, W. B., Kamiyama, K., \& Kawakami, N. (2015). Workaholism vs. work engagement: the two different predictors of future well-being and performance. International Journal of Behavioral Medicine, 22(1), 18-23. doi: https://doi. org/10.1007/s12529-014-9410-x

Siegrist, J. (2005). Social reciprocity and health: new scientific evidence and policy implications. Psychoneuroendocrinology, 30, 1033-1038. https://doi.org/10.1016/j. psyneuen.2005.03.017

Sociedad Mexicana de Psicología. (2007). Código Ético del Psicólogo (4ª . edición). México: Trillas.

Soltani, M., \& Ali, A. (2016). A cross-level model of organizational commitment antecedents. Iranian Journal of Management Studies, 9(2), 283-405. doi: 10.22059/ ijms.2016.56416

Soto, C. J. (2015). Is happiness good for your personality? Concurrent and prospective relations of the big five with subjective well? ?being. Journal of personality, 83(1), 45-55. doi: https://doi.org/10.1111/jopy.12081

Valenzuela, H., Reygadas, L., \& Cruces, F. (2015). Mi trabajo es mi vida. La incrustación de los mundos de la vida y del trabajo en empresas españolas, Revista Española de Investigaciones Sociológicas, 150, 191-210. doi: https://doi.org/10.5477/cis/ reis. 150.191

Vázquez, C., Hervás, G., Rahona, J. J., \& Gómez, D. (2009). Bienestar psicológico y salud: Aportaciones desde la Psicología Positiva. Anuario de psicología Clínica y de la Salud, 5, 15-28.

Werther, W., \& Davis, K. (2000). Administración de Personal y Recursos humanos quinta edición. México: Mc Graw Hill.

Yousef, D. A. (2016). Organizational Commitment, Job Satisfaction and Attitudes toward Organizational Change: A Study in the Local Government. International Journal of Public Administration, 1-12. doi: https://doi.org/10.1080/01900692.2 $\underline{015.1072217}$ 